\title{
Modeling of nonlinear viscous stress in encapsulating shells of lipid-coated contrast agent microbubbles
}

\author{
Alexander A. Doinikov ${ }^{a,}{ }^{*}$, Jillian F. Haac ${ }^{b}$, and Paul A. Dayton ${ }^{\mathrm{b}, \mathrm{c}}$ \\ alnstitute of Nuclear Problems, Belarus State University, 11 Bobruiskaya Street, Minsk 220030, \\ Belarus \\ bUNC-NCSU Joint Department of Biomedical Engineering, 302 Taylor Hall, CB 7575, Chapel Hill, \\ NC 27599, USA \\ CUC Davis Department of Biomedical Engineering, 1 Shields Ave, Davis, CA 95616, USA
}

\section{Abstract}

A general theoretical approach to the development of zero-thickness encapsulation models for contrast microbubbles is proposed. The approach describes a procedure that allows one to recast available rheological laws from the bulk form to a surface form which is used in a modified RayleighPlesset equation governing the radial dynamics of a contrast microbubble. By the use of the proposed procedure, the testing of different rheological laws for encapsulation can be carried out. Challenges of existing shell models for lipid-encapsulated microbubbles, such as the dependence of shell parameters on the initial bubble radius and the "compression-only" behavior, are discussed. Analysis of the rheological behavior of lipid encapsulation is made by using experimental radius-time curves for lipid-coated microbubbles with radii in the range $1.2-2.5 \mu \mathrm{m}$. The curves were acquired for a research phospholipid-coated contrast agent insonified with a 20-cycle, $3.0 \mathrm{MHz}, 100 \mathrm{kPa}$ acoustic pulse. The fitting of the experimental data by a model which treats the shell as a viscoelastic solid gives the values of the shell surface viscosity increasing from $0.30 \times 10^{-8} \mathrm{~kg} / \mathrm{s}$ to $2.63 \times 10^{-8} \mathrm{~kg} / \mathrm{s}$ for the range of bubble radii indicated above. The shell surface elastic modulus increases from $0.054 \mathrm{~N} /$ $\mathrm{m}$ to $0.37 \mathrm{~N} / \mathrm{m}$. It is proposed that this increase may be a result of the lipid coating possessing the properties of both a shear-thinning and a strain-softening material. We hypothesize that these complicated rheological properties do not allow the existing shell models to satisfactorily describe the dynamics of lipid encapsulation. In the existing shell models, the viscous and the elastic shell terms have the linear form which assumes that the viscous and the elastic stresses acting inside the lipid shell are proportional to the shell shear rate and the shell strain, respectively, with constant coefficients of proportionality. The analysis performed in the present paper suggests that a more general, nonlinear theory may be more appropriate. It is shown that the use of the nonlinear theory for shell viscosity allows one to model the "compression-only" behavior. As an example, the results of the simulation for a 2.03- $\mu$ m-radius bubble insonified with a 6-cycle, $1.8 \mathrm{MHz}, 100 \mathrm{kPa}$ acoustic pulse are given. These parameters correspond to the acoustic conditions under which the "compression-only" behavior was observed by de Jong et al. [Ultrasound Med. Biol. 33 (2007) 653656]. It is also shown that the use of the Cross law for the modeling of the shear-thinning behavior of shell viscosity reduces the variance of experimentally estimated values of the shell viscosity and its dependence on the initial bubble radius.

(C) 2008 Elsevier B.V. All rights reserved.

*Corresponding author. Tel.: +375 17 2264231; fax: +375 17 2265124. doinikov@bsu.by (A. Doinikov).

Publisher's Disclaimer: This is a PDF file of an unedited manuscript that has been accepted for publication. As a service to our customers we are providing this early version of the manuscript. The manuscript will undergo copyediting, typesetting, and review of the resulting proof before it is published in its final citable form. Please note that during the production process errors may be discovered which could affect the content, and all legal disclaimers that apply to the journal pertain. 


\section{Keywords}

Contrast agent; Shell model; Lipid coating; Compression-only behavior; Nonlinear viscosity

\section{Introduction}

Encapsulated gas microbubbles are used in ultrasound medical applications as contrast agents to enhance the acoustic contrast between blood and surrounding tissues and thereby to improve the quality of ultrasonic images [1-3]. The function of encapsulation is to stabilize microbubbles against fast dissolution and coalescence. Current available contrast agents are enclosed in a shell of albumin, polymer, or lipid. The present study focuses on lipid-shelled microbubbles.

Theoretical description of the rheological behavior of the shell material is of primary importance as it is the shell that determines many of the functional properties of contrast agent microbubbles. Much work has been done on modeling the dynamics of encapsulated microbubbles in an ultrasound field. Most of the existing shell models have the form of a modified Rayleigh-Plesset equation and assume, explicitly or implicitly, that the shell material behaves as a viscoelastic solid [4-12]. De Jong and co-workers pioneered in the development of such models [4-7], and have demonstrated the ability to accurately model experimental microbubble radius-time oscillations. In addition to these models which assume that the shell is a viscoelastic solid, there are also models that treat the encapsulation as a viscous Newtonian fluid [11,13-15] or a viscoelastic Maxwell fluid [16]. It should also be pointed out that the models proposed in $[8,10,12,13,16]$ were derived assuming the encapsulating layer to be of arbitrary thickness, and in $[12,16,17]$ the radial dynamics of encapsulated microbubbles was modeled taking account of the translational motion. The work of the preceding researches was very productive and gave an insight into many aspects of the dynamics of contrast microbubbles. However, we propose that there are still some limitations with the existing shell models. For example, current models suggest the dependence of shell parameters on the initial bubble radius, and have difficulty with recent experimental observations, such as "compression-only" behavior.

The "compression-only" behavior of phospholipid-coated bubbles was discovered by de Jong et al. [18]. In the course of an optical ultra high-speed contrast imaging study on individual SonoVue and BR-14 microbubbles, they observed that in some cases the microbubbles only compressed and hardly expanded beyond their initial diameters. De Jong et al. carried out pertinent numerical simulations using the shell model developed in [4-6] and came to the conclusion that "By comparing the experimental data with the simulations, it follows that "compression-only" behavior must be explained by a more sophisticated shell model, possibly including shell buckling and rupture." Such a model was proposed earlier by Marmottant $e t$ al. [19]. However, this model is based on a peculiar ad hoc law for surface tension and still is challenged by the observed dependence of the shell physical constants on the initial bubble radius.

The findings that the shell viscosity of phospholipid-coated microbubbles increases with the initial bubble radius were reported by Morgan et al. [20] and more recently van der Meer $e t$ al. [21] and Doinikov and Dayton [22]. Chetty et al. [23] report that the shell elasticity seems to behave similarly. In the present paper, we confirm these findings by means of our own experimental data. The result that the shell physical constants are found to be highly dependent on the initial bubble radius is of particular interest as it discloses that the current shell models meet with difficulties of fundamental nature. It is known that physical differential constants of a material must be independent of the total amount of the material or the area of its surface if 
surface physical constants are considered. Therefore it is appropriate to suggest that the said behavior of the shell viscosity and the shell elasticity is an artifact that arises from an inadequate description of the rheological nature of the encapsulating coating.

Thus, the purpose of the present study is to continue the search for new theoretical models that are able to account for the observed experimental effects. In particular, it will be shown that the inclusion of nonlinear shell viscosity allows one to model the "compression-only" behavior. It is also very important to select an appropriate rheological law describing the dependence of the shell viscosity on the shear rate. In this paper, an improvement in modeling the microbubble shell viscosity is suggested which reduces the variance of experimentally estimated values of the shell viscosity and its dependence on the initial bubble radius.

In Section 2, a general theoretical approach to the development of zero-thickness encapsulation models for contrast microbubbles is proposed. A mathematical procedure is described that allows one to recast available rheological laws from the bulk form to a surface form which is used in a modified Rayleigh-Plesset equation governing the radial dynamics of a contrast microbubble. Section 3 investigates the rheological behavior of lipid encapsulation by using experimental radius-time curves for lipid-coated microbubbles. In Section 4.1, the general nonlinear viscous theory is applied to the problem of an encapsulated bubble. The results of Section 2, 3, and 4.1 are then used in Sections 4.2 and 4.3 to model the "compression-only" and the shear-thinning behavior of lipid-coated microbubbles. The conclusions are stated briefly in Section 5 .

\section{Zero-thickness encapsulation model: General theory}

We begin with the derivation of a general equation for the radial dynamics of a thin-shelled microbubble that provides a way of testing different rheological laws for encapsulation. The most theoretically justified equation for the description of the radial dynamics of an encapsulated bubble was derived by Roy, Church, and Calabrese [13]. Later on, Church repeated the derivation in his well-known paper [8], therefore that equation is usually referred to as Church's equation. It can be written as follows

$$
\begin{aligned}
& R_{1} \ddot{R}_{1}\left[1+\left(\frac{\rho_{L}}{\rho_{S}}-1\right) \frac{R_{1}}{R_{2}}\right]+\frac{3}{2} \dot{R}_{1}^{2}\left[1+\left(\frac{\rho_{L}}{\rho_{S}}-1\right)\left(\frac{4 R_{2}^{3}-R_{1}^{3}}{3 R_{2}^{3}}\right) \frac{R_{1}}{R_{2}}\right] \\
= & \frac{1}{\rho_{S}}\left[P_{g 0}\left(\frac{R_{10}}{R_{1}}\right)^{3 \gamma}-\frac{2 \sigma_{1}}{R_{1}}-\frac{2 \sigma_{2}}{R_{2}}-4 \eta_{L} \frac{R_{1}^{2} \dot{R}_{1}}{R_{2}^{3}}-P_{0}-P_{a c}(t)-S\right],
\end{aligned}
$$

where $R_{1}(t)$ and $R_{2}(t)$ are the inner and the outer radii of the encapsulating shell, respectively, the overdot denotes the time derivative, $\rho_{L}$ and $\rho_{S}$ are the equilibrium densities of the surrounding liquid and the shell, respectively, $P_{g 0}$ is the equilibrium gas pressure within the bubble, $\gamma$ is the ratio of specific heats of the gas, $R_{10}$ and $R_{20}$ are the inner and the outer radii of the shell at rest, $\sigma_{1}$ and $\sigma_{2}$ are the surface tension coefficients for the gas-shell and the shellliquid interfaces, respectively, is the $\eta_{L}$ shear viscosity of the liquid, $P_{0}$ is the hydrostatic pressure in the liquid, and $P_{a c}(t)$ is the driving acoustic pressure at the location of the bubble. The effect of encapsulation is described by the term $\mathrm{S}$ which is given by

$$
S=-3 \int_{R_{1}}^{R_{2}} \frac{\tau_{r r}(r, t)}{r} d r,
$$

where $r$ is the radial coordinate of a spherical coordinate system with the origin at the center of the bubble, and $\tau_{r r}(r, t)$ is the radial component of the stress deviator of the shell. 
Equation (1) is valid for bubbles with a shell of finite thickness. However, most types of contrast agents have very thin shells whose thickness is much less than the bubble radius. This is especially true for lipid-coated bubbles. For such bubbles, (1) is redundant from the numerical point of view, and going to the limit of thin shell is worthwhile. In the limit of thin shell, (1) reduces to

$$
R \ddot{R}+\frac{3}{2} \dot{R}^{2}=\frac{1}{\rho_{L}}\left[P_{g 0}\left(\frac{R_{0}}{R}\right)^{3 \gamma}-\frac{2 \sigma}{R}-4 \eta_{L} \frac{\dot{R}}{R}-P_{0}-P_{a c}(t)-S\right],
$$

where $R(t)$ denotes the radius of the gas-liquid interface, $R_{0}$ is the resting value of $R(t)$, and $\sigma$ is the surface tension at the gas-liquid interface. In the same limit, the term $S$ becomes

$$
S=-\left.\frac{3 \varepsilon}{R} \tau_{r r}(r, t)\right|_{r=R},
$$

with $\varepsilon$ denoting the shell thickness. Substituting different expressions for $\tau_{r r}$ into (4), one can apply different rheological laws to the encapsulating shell.

The advantage of (4) is that it shows how existing constitutive equations for the stress tensor $\tau_{i j}$, which are normally specified in the bulk form, can be recast to a surface form which is required in (3). As an example, let us consider the Kelvin-Voigt constitutive equation which describes a viscoelastic solid and is given by [24]

$$
\tau_{r r}=2 \eta_{s} \frac{\partial v}{\partial r}+2 \mu_{s} \frac{\partial u}{\partial r}
$$

where $v(r, t)$ is the radial component of the particle velocity inside the shell, $\eta_{S}$ is the bulk shear viscosity of the shell (in Pa.s), $u(r, t)$ is the radial displacement inside the shell, and $\mu_{S}$ is the bulk shear modulus of the shell (in Pa). In the case of spherical symmetry, assuming the shell material to be incompressible, one has [8]

$$
v=\frac{R^{2} \dot{R}}{r^{2}}, \quad u=\frac{R^{2}}{r^{2}}\left(R-R_{e}\right)
$$

where $R_{e}$ is the unstrained equilibrium position of the shell. For thin-shelled microbubbles, $R_{e}$ can be set equal to $R_{0}$ without a tangible loss in accuracy. Substitution of (6) into (5) and (4) yields

$$
S=S_{v i s}+S_{e l}=4 k_{S} \frac{\dot{R}}{R^{2}}+4 \chi \frac{R_{e}}{R}\left(\frac{1}{R_{e}}-\frac{1}{R}\right)
$$

Here we have also set $\kappa_{S}=3 \varepsilon \eta_{s}$ and $\chi=3 \varepsilon \gamma_{s}$, so now $\kappa_{S}$ and $\chi$ denote the shell surface viscosity (in $\mathrm{kg} / \mathrm{s}$ ) and the shell surface elastic modulus (in N/m), respectively. It is easy to see that, when (7) is substituted into (3), we virtually obtain the shell model proposed by de Jong et al. [6].

In what follows, it will be assumed that the term $S$ can be divided into viscous and elastic parts as in (7). We introduce this assumption in order to adhere to simple rheological models as far as possible. However, for the completeness of information, we would like to note that 
rheological laws do not all allow such a separation. For example, on the assumption that the shell material is a viscoelastic fluid rather than a viscoelastic solid, the Maxwell constitutive equation can be used [16],

$$
\tau_{r r}+\lambda \frac{\partial \tau_{r r}}{\partial t}=2 \eta_{s} \frac{\partial v}{\partial r}
$$

where $\lambda$ designates the relaxation time of the shell. In contrast to (5), (8) allows elastic stresses to relax, but it does not allow one to separate a purely elastic part of the stress. At the same time, the viscous terms in (5) and (8) are of the same form. This is also true for many other rheological laws. There are points at which theory can go different ways. The assumption that the term $S$ can be divided into viscous and elastic parts is one of such points. At this point, one can choose a more complicated rheological model, if necessary.

\section{Rheology of lipid coating}

The purpose of this section is to explain why the existing shell models cause the shell physical constants to be dependent on the initial bubble radius. By way of example let us consider the widely used shell model proposed by de Jong et al. [6]. It can be represented as [21]

$$
S=4 \kappa_{S} \frac{\dot{R}}{R^{2}}+4 \chi\left(\frac{1}{R_{0}}-\frac{1}{R}\right) .
$$

In a qualitative sense, the de Jong model is identical to the Kelvin-Voigt shell model, given by (7), and the Sarkar shell model [11].

To evaluate the shell parameters appearing in (9), experimental radius-time curves for 20 microbubbles of various radii were used. Experimental radius-time curves of microbubbles oscillating in response to an acoustic pulse were acquired with an Imacon 468 (DRS Hadland) high speed imaging system coupled with an Olympus IX71 inverted microscope. An arbitrary waveform generator (AWG2021, Tektronix) and radiofrequency amplifier (3200L, ENI) excited a single-element transducer (V305, Panametrics) to produce ultrasound pulses. This experimental system has been described in detail previously [17,20]. Spatial calibrations were performed using a calibrated stage reticle (Edmund Optics) and acoustic calibration and alignment was performed using a needle hydrophone (PZT-0400, Onda Corp.).

The curves were acquired for a phospholipid-coated, perfluorocarbon-core contrast agent formulated as described previously [25], insonified with a 20-cycle, $3.0 \mathrm{MHz}, 100 \mathrm{kPa}$ acoustic pulse. The shell parameters were evaluated by fitting simulated radius-time curves to the experimental data by the least squares method using the program package Mathematica. The best-fit values of $\kappa_{S}$ and $\chi$ versus the initial bubble radius are shown by circles in Figs. 1a and $1 \mathrm{~b}$, respectively. Each circle represents the best fit for one experimental radius-time curve. The solid lines show the linear regression for the best-fit values of $\kappa_{S}$ and $\chi$. It is seen that both the shell viscosity and the shell elasticity demonstrate a conspicuous increase with the initial bubble radius: $\left(\kappa_{S}\right)_{\max } /\left(\kappa_{S}\right)_{\min } \approx 8.7, \chi_{\max } / \chi_{\min } \approx 6.8$.

Van der Meer et al. [21] hypothesized that the observed dependence of the shell viscosity on the initial bubble radius is a consequence of the shell viscosity being dependent on the shear rate, which is proportional to $\dot{R} / R$ in our case. Figure 2a confirms this hypothesis. It shows the dependence between the shell viscosity and the maximum shear rate for the experimental data considered. The shell viscosity was evaluated by the de Jong model, as in Fig. 1a, and the maximum shear rate, which is estimated as the maximum value of $\dot{R} / R$, was calculated directly 
from the experimental radius-time curves. One can see that the shell viscosity decreases as the shear rate increases. This type of rheological behavior is known as shear thinning. Figure 2a reveals thus that the lipid coating appears to be a shear-thinning material. This result suggests that the dependence of $\kappa_{S}$ on $R_{0}$, which is shown by the de Jong model, arises because the linear viscoelastic theory may not account for the full complexity of the rheological nature of the lipid shell. For this reason, we suggest that the viscosity of the lipid shell may need to be described by a more complex theory than that underlying the existing shell models.

A more physical picture of the behavior of the shell elasticity can be obtained by plotting $\chi$ as a function of the deformation strength. As a measure of the deformation strength, the quantity $\left(R_{\max }-R_{0}\right) / R_{0}$ can be taken. The plot of $\chi$ versus the estimated deformation strength is shown in Fig. 2b. The values of $\chi$ were evaluated by the de Jong model, as in Fig. 1b, and the quantity $\left(R_{\max }-R_{0}\right) / R_{0}$ was calculated directly from the experimental radius-time curves. Figure $2 \mathrm{~b}$ reveals that the shell elasticity decreases as the deformation strength increases. This rheological effect is known as strain softening. Thus there is a reason to believe that the lipid coating has the properties of both shear-thinning and strain-softening material. Therefore we hypothesize that the theory for the shell elasticity may need to be revised as well.

In the existing shell models, the viscous and the elastic shell terms have the simplest possible form, namely, the linear form. This linear theory assumes that the viscous and the elastic stresses acting inside the lipid shell are proportional to the shell shear rate and the shell strain, respectively, with constant coefficients of proportionality $\kappa_{S}$ and $\chi$. The analysis presented here shows that a more general, nonlinear theory for both the viscous and the elastic shell terms is required. In connection with this inference, a recent paper by Tsiglifis and Pelekasis [26] should be mentioned, where an attempt is made to use the nonlinear elastic laws by Mooney-Rivlin and Skalak to model some aspects of the dynamics of encapsulated bubbles. In the present paper, we focus on the nonlinear theory for viscous shell stress.

\section{Nonlinear viscosity}

In this section, the general nonlinear viscous theory is applied to the problem of an encapsulated bubble. The results are then used to model the "compression-only" and the shear-thinning behavior of lipid-coated microbubbles.

\subsection{General theory}

In the general case, the relationship between the viscous stress tensor and the rate-of-strain tensor is written as [27]

$$
\tau_{i j}^{(v i s)}=F\left(V_{i j}\right),
$$

where $\tau_{i j}^{(v i s)}$ is the viscous stress tensor, $F$ is an arbitrary function, and $V_{i j}$ is the rate-of-strain tensor. This equation is known as the Stokes hypothesis [27]. Actually, the viscous stress tensor can be also dependent on other kinematic quantities in addition to the rate-of-strain tensor, such as the deformation acceleration [27]. However, if we employ simple models as far as possible, (10) should be considered as a next step as compared to the linear viscous term in (7) and (9).

In the mathematical basis of rheology [27], it is proven that, according to the principle of material objectivity, if the material is isotropic and incompressible, (10) must be of the following form: 


$$
\tau_{i j}^{(v i s)}=2 \eta_{1}\left(I_{2}, I_{3}\right) V_{i j}+4 \eta_{2}\left(I_{2}, I_{3}\right) V_{i k} V_{k j},
$$

where summation over double indices is implied, and $\eta_{1}$ and $\eta_{2}$ are arbitrary functions of the second and the third invariants of the rate-of-strain tensor $V_{i j} . I_{2}$ and $I_{3}$ can be specified as

$$
I_{2}=\operatorname{Tr}\left(V_{i k} V_{k j}\right)=V_{i k} V_{k i}, \quad I_{3}=\operatorname{det}\left(V_{i j}\right)=V_{i k} V_{k j} V_{j i} .
$$

Equation (11) is known as the constitutive equation for a Reiner-Rivlin fluid [27]. Newtonian fluids are a particular case of Reiner-Rivlin fluids for which $\eta_{1}=$ const and $\eta_{2}=0$.

For an encapsulated bubble, in view of spherical symmetry, (11) takes the form

$$
\tau_{r r}^{(v i s)}=2 \eta_{1}\left(I_{2}, I_{3}\right) V_{r r}+4 \eta_{2}\left(I_{2}, I_{3}\right)\left(V_{r r}\right)^{2},
$$

with

$$
V_{r r}=\frac{\partial v}{\partial r}=-\frac{2 R^{2} \dot{R}}{r^{3}}, I_{2}=\frac{6 R^{4} \dot{R}^{2}}{r^{6}}, \quad I_{3}=-\frac{2 R^{6} \dot{R}^{3}}{r^{9}} .
$$

Let us now assume that the total stress tensor is given by an equation similar to the KelvinVoigt constitutive equation, equation (5),

$$
\tau_{r r}=\tau_{\mathrm{rr}}^{(v i s)}+2 \mu_{s} \frac{\partial u}{\partial r}
$$

However, unlike (5), the viscous part is now specified by (13). Then, using (4) and (6), we obtain the term $S$ of (3) to be

$$
\begin{gathered}
S=S_{v i s}+S_{e l}=12 \varepsilon \frac{\dot{R}}{R^{2}}[ \\
\left.=\left.\eta_{1}\left(I_{2}, I_{3}\right)\right|_{r=R}-\left.4 \frac{\dot{R}}{R} \eta_{2}\left(I_{2}, I_{3}\right)\right|_{r=R}\right]+4 \chi \frac{R_{e}}{R}\left(\frac{1}{R_{e}}-\frac{1}{R}\right) \\
=4 \kappa(\dot{R} / R) \frac{\dot{R}}{R^{2}}+4 \chi \frac{R_{e}}{R}\left(\frac{1}{R_{e}}-\frac{1}{R}\right),
\end{gathered}
$$

where $\kappa$ is a function of the quantity $\dot{R} / R$ which can be treated as the shear rate of the shell. In the following calculations, it will be assumed that $R_{e}=R_{0}$.

Although our derivation led to the final result that could have been guessed and expected $a b$ initio, the significance of this derivation is that it argues strictly for the physically correct form of the nonlinear viscous term. It should be emphasized, however, that the form of the viscous term in (16) is just a particular case resulting from the fact that the viscous and elastic parts of the total stress tensor are separate. For example, in the case of the Maxwell equation, equation (8), the situation is different. It is known that with the linear viscous term, (8) can be readily solved [16]. If, however, the linear viscous term is replaced with (13), it is impossible to solve (8) if we do not know explicit expressions for the functions $\eta_{1}$ and $\eta_{2}$.

Turning back to (16), it should be recognized that theory cannot indicate a more exact form of the function $\kappa(\dot{R} / R)$ in the case under consideration. The literature on rheology shows that the 
only further way to determine an explicit form of $\kappa(\dot{R} / R)$ is to select (guess) a suitable analytical function using experimental data.

\subsection{Modeling of "compression-only" behavior}

As mentioned above, it has been found experimentally that many phospholipid-coated contrast agents show "compression-only" behavior, where the microbubbles compress much more strongly than they expand [18]. As a result, the relative ratio of expansion to compression, E/ $C=\mid\left(R_{\max }-R_{0}\right) /\left(R_{0}-R_{\min }\right) \mathrm{I}$, may be as small as 0.5 [28]. The Kelvin-Voigt shell model, equation (7), as well as the de Jong shell model, equation (9), are challenged by this phenomenon because they predict $E / C$ values to be close to, or above unity [28]. In [18], it is hypothesized that the "compression-only" behavior may be a result of shell buckling. We will show here that this effect can be modeled in terms of nonlinear shell viscosity. It should be noted that, even if the "compression-only" behavior is really caused by shell buckling, formally mathematically, this effect can be modeled as a change in the shell properties, or, in other words, as a specific behavior of the shell, assuming that the shape of the bubble remains spherical. This is acceptable because in fact we are interested in the scattered echo from the bubble rather than the radial bubble dynamics per se. Therefore, if we are able to approximate the scattered signal from a buckled bubble as if it were a signal from a spherical bubble with specific shell properties, it makes no difference whether the real bubble, as a source of the signal, is buckled or not.

Let us assume that the function $\kappa(\dot{R} / R)$ in (16) takes the form

$$
\kappa(\dot{R} / R)=\kappa_{0}+\kappa_{1} \frac{\dot{R}}{R}
$$

where $\kappa_{0}$ and $\kappa_{1}$ are constants. Choosing this simple form for the function $\kappa(\dot{R} / R)$, we follow the conventional approach which is used in physics when the form of a sought-for function is unknown. In such cases, it is assumed that the function can be expanded in a Taylor series and then, as a first approximation, the first two terms of the expansion are retained. For $\kappa_{1}=0,(16)$ is identical to the Kelvin-Voigt model. As an example, Fig. 3 shows two radius-time curves that were calculated by (3), (16), and (17) for a bubble with $R_{0}=2.03 \mu \mathrm{m}$, insonified with a 6cycle, $1.8 \mathrm{MHz}, 100 \mathrm{kPa}$ acoustic pulse. These acoustic parameters correspond to Fig. 2 of [18]. The curve in Fig. 3a was calculated at $\chi=0.5 \mathrm{~N} / \mathrm{m}, 1.5 \times 10^{-8} \mathrm{~kg} / \mathrm{s}$, and $\kappa_{1}=0$, i.e., that is a curve given by the Kelvin-Voigt model. The curve in Fig. $3 \mathrm{~b}$ was calculated for the same parameters except that $\kappa_{1}=1.0 \times 10^{-14} \mathrm{~kg}$. One can see that this curve does show a response that is quite similar to the "compression-only" behavior, with sharp edges in the compression phase as reported by de Jong et al. [18].

Figure 4 represents results given by the Kelvin-Voigt model and the model with equation (17) when a simulated radius-time curve is fitted to one of our experimental radius-time curves showing "compression-only" behavior. The experimental data were acquired as described above in Section 3. The fitting was done by the least squares method using the program package Mathematica. An example of the experimental radius-time curve is displayed in Fig. 4a. The curve was acquired for a phospholipid-coated bubble with $R_{0} \approx 1.4 \mu \mathrm{m}$. The bubble was insonified with a 20-cycle, $3.0 \mathrm{MHz}, 100 \mathrm{kPa}$ acoustic pulse. Figure $4 \mathrm{~b}$ shows the best fit that was obtained by the least squares method using the Kelvin-Voigt model. The solid line represents the simulated radius-time curve and circles indicate the experimentally measured points. Figure $4 \mathrm{~b}$ corresponds to the part of the experimental curve in Fig. 4 a between 3 and 5 $\mu \mathrm{s}$. The best fit given by the model with equation (17) is shown in Fig. 4c. One can see that the application of (17) improves considerably agreement between the theoretical curve and the experimental data. 


\subsection{Modeling of shear-thinning behavior}

It was shown in the preceding subsection that (17) makes it possible to simulate "compressiononly" behavior. However, the dependence of $\kappa_{0}$ and $\kappa_{1}$ on the initial bubble radius still persists. This is confirmed by Fig. 5, which presents the best-fit values of $\kappa_{0}$ and $\kappa_{1}$ versus the initial bubble radius for the same experimental data as in section 3 . The best-fit values of $\kappa_{0}$ and $\kappa_{1}$ are shown by circles in Figs. 5a and 5b, respectively. The best-fit values of $\chi$, Fig. 5c, are presented for completeness. Note that Fig. 5a is almost identical to Fig. 1a. This means that the presence of the term $\kappa_{1} \dot{R} / R$ in (17) does not virtually change the constant component of the shell viscosity, $\kappa_{0}$, as compared to the shell viscosity of the de Jong and the Kelvin-Voigt models, $\kappa_{s}$. This follows if we take into consideration that the term $\kappa_{1} \dot{R} / R$ is responsible for "compression-only" behavior, while $\kappa_{0}$ describes the behavior of lipid as a material. If that is true, the spread of the values of $\kappa_{1}$ in Fig. $5 \mathrm{~b}$ can be explained as follows. It is hypothesized in [18] that "compression-only" behavior is a result of initial shell buckling. In its turn, the degree of initial buckling for a particular bubble is likely to be a result of random factors so that bubbles of the same size can have a different degree of initial buckling. Therefore the disordered spread of the values of $\kappa_{1}$ can be expected. This is not the case, however, for $\kappa_{0}$, which is assumed to be a constant of lipid as a material, and therefore the dependence of this constant on the initial bubble radius requires a further consideration.

Considering Fig. 2a, we should seek a law for $\kappa_{0}$ that is to describe shear-thinning behavior. Adhering to simple models as before, let us try the following equation:

$$
\kappa(\dot{R} / R)=\frac{\kappa_{0}}{1+\alpha|R| / R}+\kappa_{1} \frac{\dot{R}}{R} .
$$

The first term on the right-hand side of this equation is a particular case of the Cross law which is widely used in rheology to model shear-thinning behavior [29]. With equation (18), setting also $\alpha=4 \mu$ s, the fitting of the same experimental data as in Figs. 1 and 5 gives the values of $\kappa_{0}$ and $\kappa_{1}$ shown in Fig. 6. As might be expected, the spread of the values of $\kappa_{1}$ in Fig. 6b as compared to Fig. $5 \mathrm{~b}$ decreases insignificantly. While the spread of the values of $\kappa_{0}$ becomes noticeably smaller than in Fig. 5a, and we now have $\left(\kappa_{0}\right)_{\max }\left(\kappa_{0}\right)_{\min } \approx 2.4$ instead of $\left(\kappa_{0}\right)_{\text {max }}$ $\left(\kappa_{0}\right)_{\min } \approx 8$ as in Fig. 5a.

These results show that by considering the lipid shell as both a shear-thinning and strainsoftening material, improved agreement between experimental data and theory can be achieved. Without fully accounting for the complex rheology of the lipid shell, interpretation of the experimental data may be misleading and can imply an unnatural dependence of the shell physical constants on the bubble size.

\section{Conclusions}

In the present paper, we suggest that lipid coatings exhibit the properties of both shear-thinning and strain-softening material. If lipid-shelled microbubbles do indeed behave with these complicated rheological properties, then existing shell models, which are based on the linear theory of viscosity and elasticity, will be challenged to describe the dynamics of lipid encapsulation. It is shown that by using the nonlinear viscous theory, one can model the "compression-only" behavior of lipid-coated microbubbles, the dependence of the shell viscosity on the shear rate, and reduce the dependence of the shell viscous coefficient on the initial bubble radius. We encourage our colleagues in the field that have access to experimental radius-time curves to further evaluate our suggestions of lipid-encapsulated microbubbles as both shear-thinning and strain-softening. 


\section{Acknowledgments}

A. A. D. wishes to acknowledge the financial support of the International Science and Technology Center (ISTC) under Contract No. B-1213. P. A. D. and J. F. H. provided experimental data for this manuscript, and are supported by the NIH Roadmap for Medical Research, EB005325. We thank Katherine Ferrara for use of the high-speed imaging system.

\section{References}

1. Becher, H.; Burns, PN. Handbook of Contrast Echocardiography: Left ventricular function and myocardial perfusion. Vol. 1. Springer Verlag; New York: 2000. p. 1-44.

2. Goldberg, BB.; Raichlen, JS.; Forsberg, F., editors. Ultrasound Contrast Agents: Basic Principles and Clinical Applications. Martin Dunitz; London: 2001.

3. Szabo, T. Diagnostic Ultrasound Imaging: Inside Out. Academic; New York: 2004.

4. de Jong N, Hoff L, Skotland T, Bom N. Absorption and scatter of encapsulated gas filled microspheres: Theoretical consideration and some measurements. Ultrasonics 1992;30:95-103. [PubMed: 1557838]

5. de Jong N, Hoff L. Ultrasound scattering of Albunex microspheres. Ultrasonics 1993;31:175-181. [PubMed: 8484195]

6. de Jong N, Cornet R, Lancée CT. Higher harmonics of vibrating gas-filled microspheres. Part one: simulations. Ultrasonics 1994;32:447-453.

7. Frinking PJA, de Jong N. Acoustic modeling of shell-encapsulated gas bubbles. Ultrasound Med Biol 1998;24:523-533. [PubMed: 9651962]

8. Church CC. The effect of an elastic solid surface layer on the radial pulsations of gas bubbles. J Acoust Soc Am 1995;97:1510-1521.

9. Hoff L, Sontum PC, Hovem JM. Oscillations of polymeric microbubbles: Effect of the encapsulating shell. J Acoust Soc Am 2000;107:2272-2280. [PubMed: 10790053]

10. Khismatullin DB, Nadim A. Radial oscillations of encapsulated microbubbles in viscoelastic liquids. Phys Fluids 2002;14:3534-3557.

11. Sarkar K, Shi WT, Chatterjee D, Forsberg F. Characterization of ultrasound contrast microbubbles using in vitro experiments and viscous and viscoelastic interface models for encapsulation. $\mathrm{J}$ Acoust Soc Am 2005;118:539-550. [PubMed: 16119373]

12. Doinikov AA, Dayton PA. Spatio-temporal dynamics of an encapsulated gas bubble in an ultrasound field. J Acoust Soc Am 2006;120:661-669.

13. Roy, RA.; Church, CC.; Calabrese, A. Cavitation produced by short pulses of ultrasound. In: Hamilton, MF.; Blackstock, DA., editors. Frontiers of Nonlinear Acoustics: Proceedings of 12th ISNA. El-sevier; London: 1990. p. 476-481.

14. Allen JS, May DJ, Ferrara KW. Dynamics of therapeutic ultrasound contrast agents. Ultrasound Med Biol 2002;28:805-816. [PubMed: 12113793]

15. Chatterjee D, Sarkar K. A Newtonian rheological model for the interface of microbubble contrast agents. Ultrasound Med Biol 2003;29:1749-1757. [PubMed: 14698342]

16. Doinikov AA, Dayton PA. Maxwell rheological model for lipid-shelled ultrasound microbubble contrast agents. J Acoust Soc Am 2007;121:3331-3340. [PubMed: 17552685]

17. Dayton PA, Allen JS, Ferrara KW. The magnitude of radiation force on ultrasound contrast agents. J Acoust Soc Am 2002;112:2183-2192. [PubMed: 12430830]

18. de Jong N, Emmer M, Chin CT, Bouakaz A, Mastik F, Lohse D, Versluis M. "Compression-only" behavior of phospholipid-coated contrast bubbles. Ultrasound Med Biol 2007;33:653-656. [PubMed: 17320268]

19. Marmottant P, van der Meer S, Emmer M, Versluis M, de Jong N, Hilgenfeldt S, Lohse D. A model for large amplitude oscillations of coated bubbles accounting for buckling and rupture. J Acoust Soc Am 2005;118:3499-3505.

20. Morgan KE, Allen JS, Dayton PA, Chomas JE, Klibanov AL, Ferrara KW. Experimental and theoretical evaluation of microbubble behavior. Effect of transmitted phase and bubble size, IEEE Trans Ultrason Ferroelect Freq Contr 2000;47:1494-1509. 
21. van der Meer SM, Dollet B, Voormolen MM, Chin CT, Bouakaz A, de Jong N, Versluis M, Lohse D. Microbubble spectroscopy of ultrasound contrast agents. J Acoust Soc Am 2007;121:648-656. [PubMed: 17297818]

22. Doinikov, AA.; Dayton, PA. Nonlinear dynamics of lipid-shelled ultrasound microbubble contrast agents. In: Mammoli, AA.; Brebbia, CA., editors. Computational Methods in Multiphase Flow IV. WIT press; Southampton, UK: 2007. p. 261-270.

23. Chetty, K.; Sennoga, CA.; Hainal, JV.; Eckersley, RJ.; Stride, E. Proceedings of the 2006 IEEE International Ultrasonics Symposium. IEEE; Vancouver, Canada: 2006. High speed optical observations and simulation results of lipid based microbubbles at low insonation pressures; $\mathrm{p}$. 1354-1357.

24. Meyers, MA.; Chawla, KK. Mechanical Behavior of Materials. Vol. Sec. 13.10. Prentice-Hall; 1999. p. $570-580$.

25. Zhao S, Borden MA, Bloch SH, Kruse D, Ferrara KW, Dayton PA. Radiation-force assisted targeting facilitates ultrasonic molecular imaging. Mol Imaging 2004;3:135-148. [PubMed: 15530249]

26. Tsiglifis K, Pelekasis NA. Nonlinear radial oscillations of encapsulated microbubbles subject to ultrasound: The effect of membrane constitutive law. J Acoust Soc Am 2008;123:4059-4070. [PubMed: 18537358]

27. Astarita, G.; Marrucci, G. Principles of Non-Newtonian Fluid Mechanics. Vol. Chapter 2. McGrawHill; London: 1974. p. 43-77.

28. Emmer M, van Wamel A, Goertz DE, de Jong N. The onset of microbubble vibration. Ultrasound Med Biol 2007;33:941-949. [PubMed: 17451868]

29. Cross MM. Rheology of non-Newtonian fluids: A new flow equation for pseudoplastic systems. J Colloid Science 1965;20:417-437. 

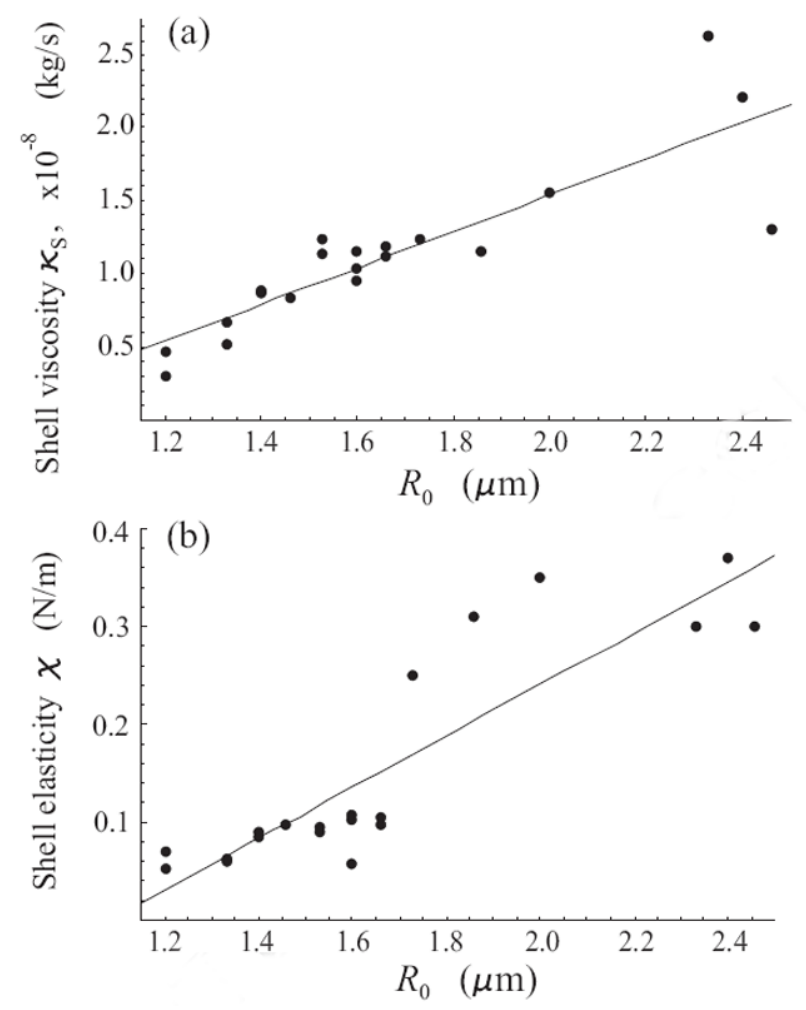

Fig. 1.

Best-fit values of the shell viscosity $\kappa_{s}$ and the shell elasticity $\chi$ versus the initial bubble radius. Bubbles are insonified with a 20 -cycle, $3.0 \mathrm{MHz}, 100 \mathrm{kPa}$ acoustic pulse. The values of $\kappa_{s}$ and $\chi$ were evaluated by the de Jong shell model. Each circle represents the best fit for one experimental radius-time curve. 

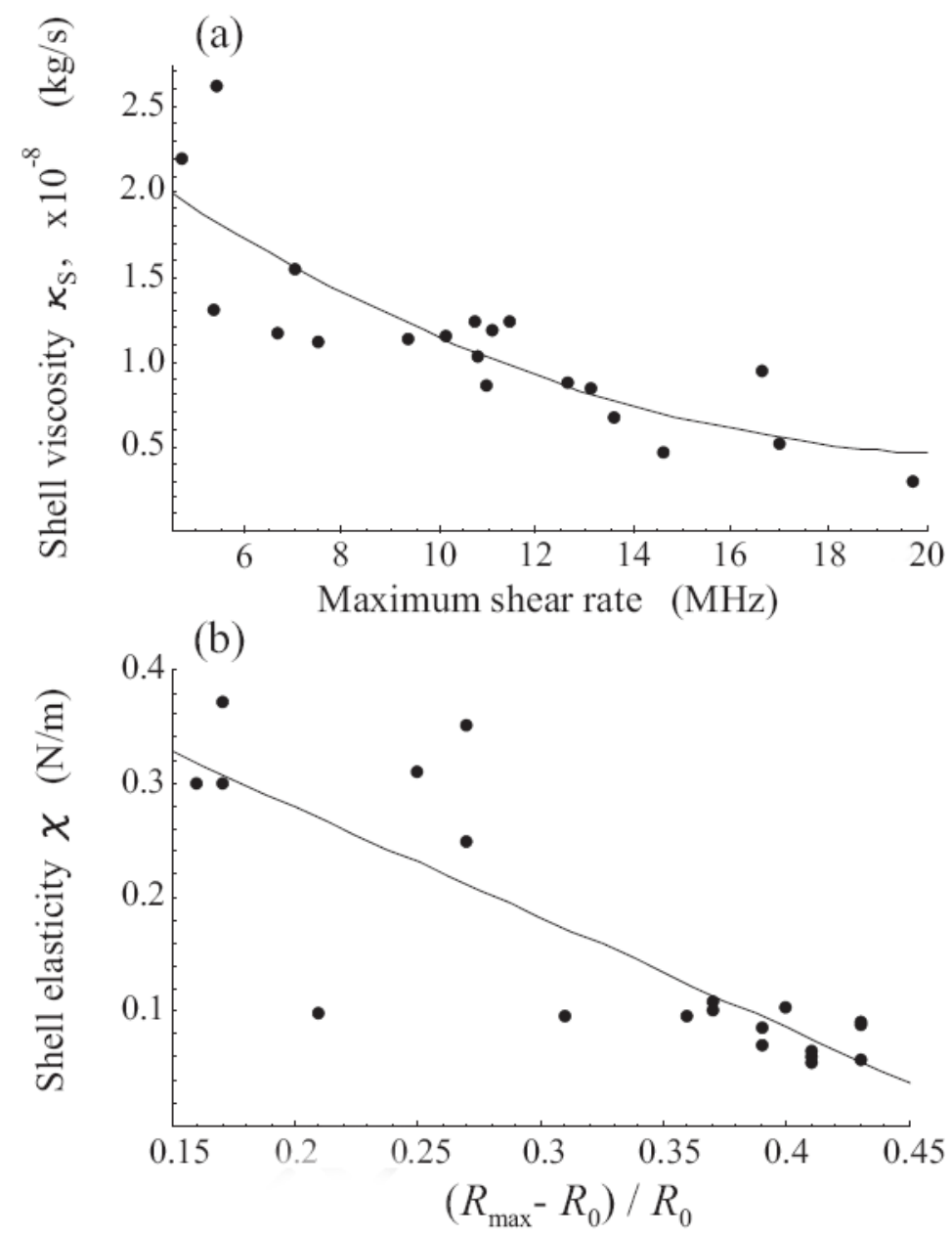

Fig. 2.

(a) Shell viscosity versus maximum shear rate. (b) Shell elasticity versus deformation strength. The values of $\kappa_{\mathrm{s}}$ and $\chi$ were evaluated by the de Jong shell model. The maximum shear rate and the deformation strength were calculated directly from experimental radius-time curves. 

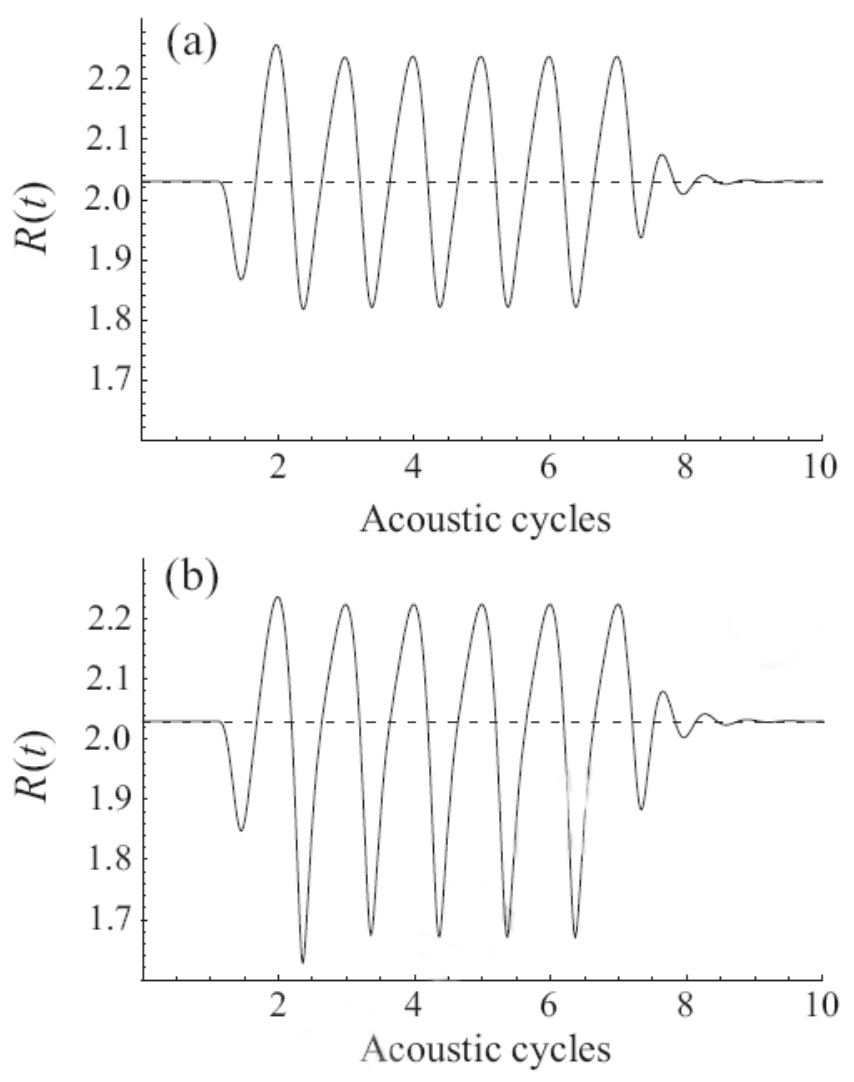

Fig. 3.

Simulated radius-time curves for a 2.03- $\mu$ m-radius encapsulated bubble insonified with a 6cycle, $1.8 \mathrm{MHz}, 100 \mathrm{kPa}$ acoustic pulse. (a) The Kelvin-Voigt shell model. (b) The model with the shell viscosity specified by equation (17). 
(a)



(b)

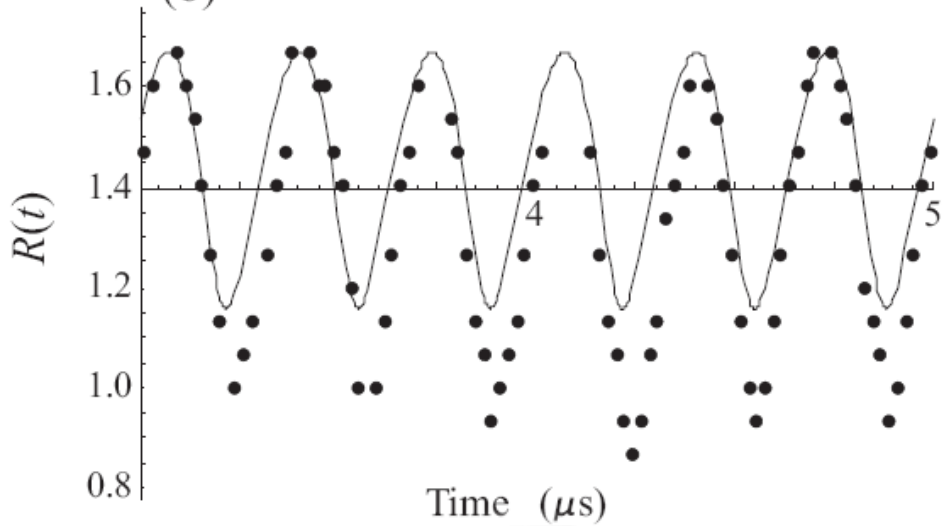

(c)

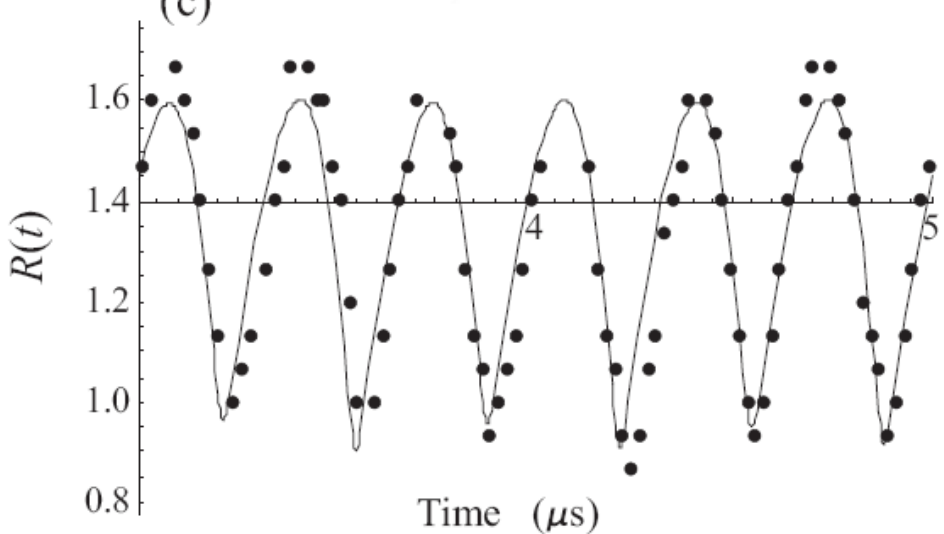

Fig. 4.

Fitting of an experimental radius-time curve showing "compression-only" behavior. (a) The experimental radius-time curve for a $1.4-\mu \mathrm{m}$-radius bubble with phospholipid coating. The excitation is a 20 -cycle, $3.0 \mathrm{MHz}, 100 \mathrm{kPa}$ acoustic pulse. (b) The best fit given by the KelvinVoigt shell model. (c) The best fit given by the model with the shell viscosity specified by equation (17). 

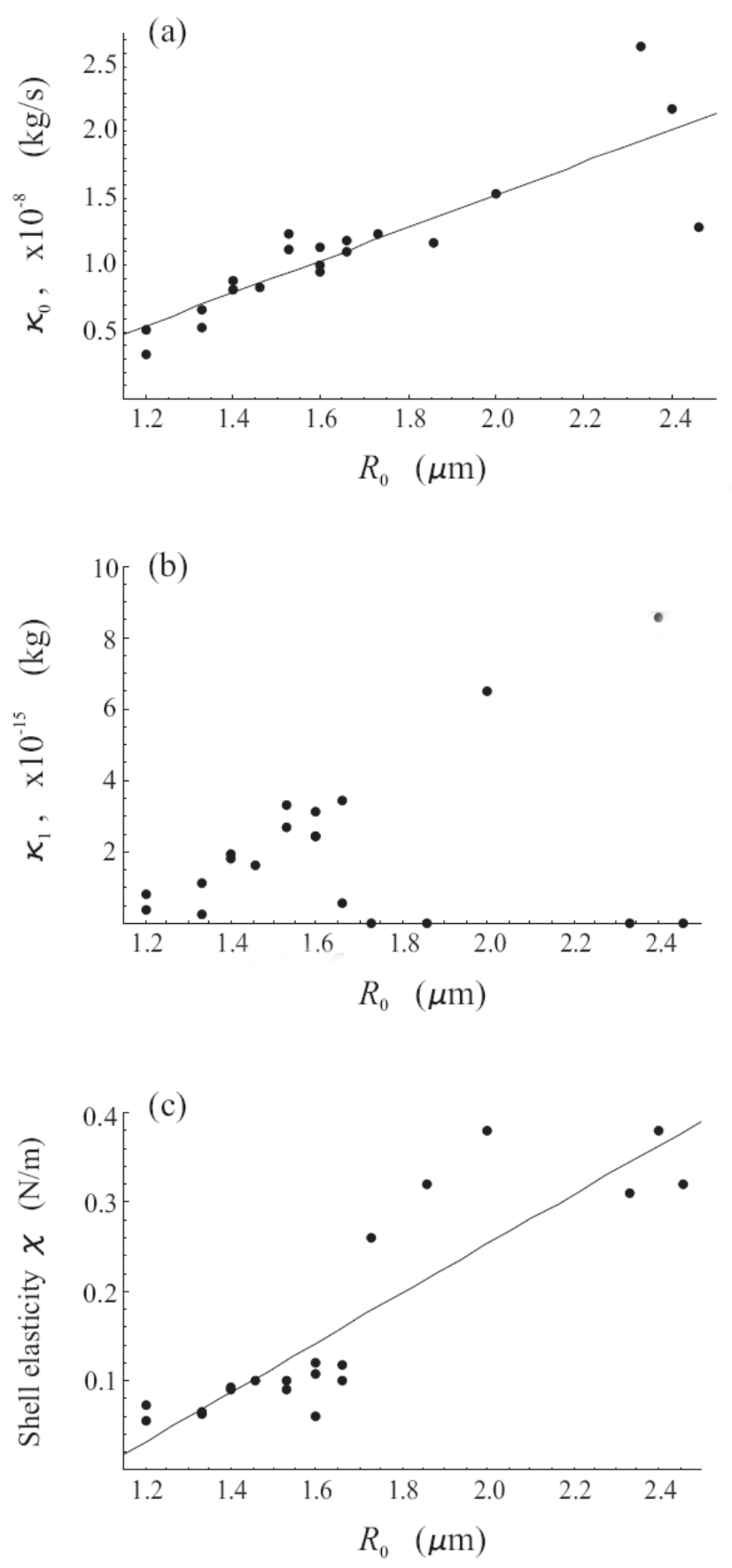

Fig. 5.

Best-fit values of the shell viscous coefficients $\kappa_{0}$ and $\kappa_{1}$, and the shell elasticity $\chi$ versus the initial bubble radius. The values of the shell parameters were evaluated by using (16) and (17). 

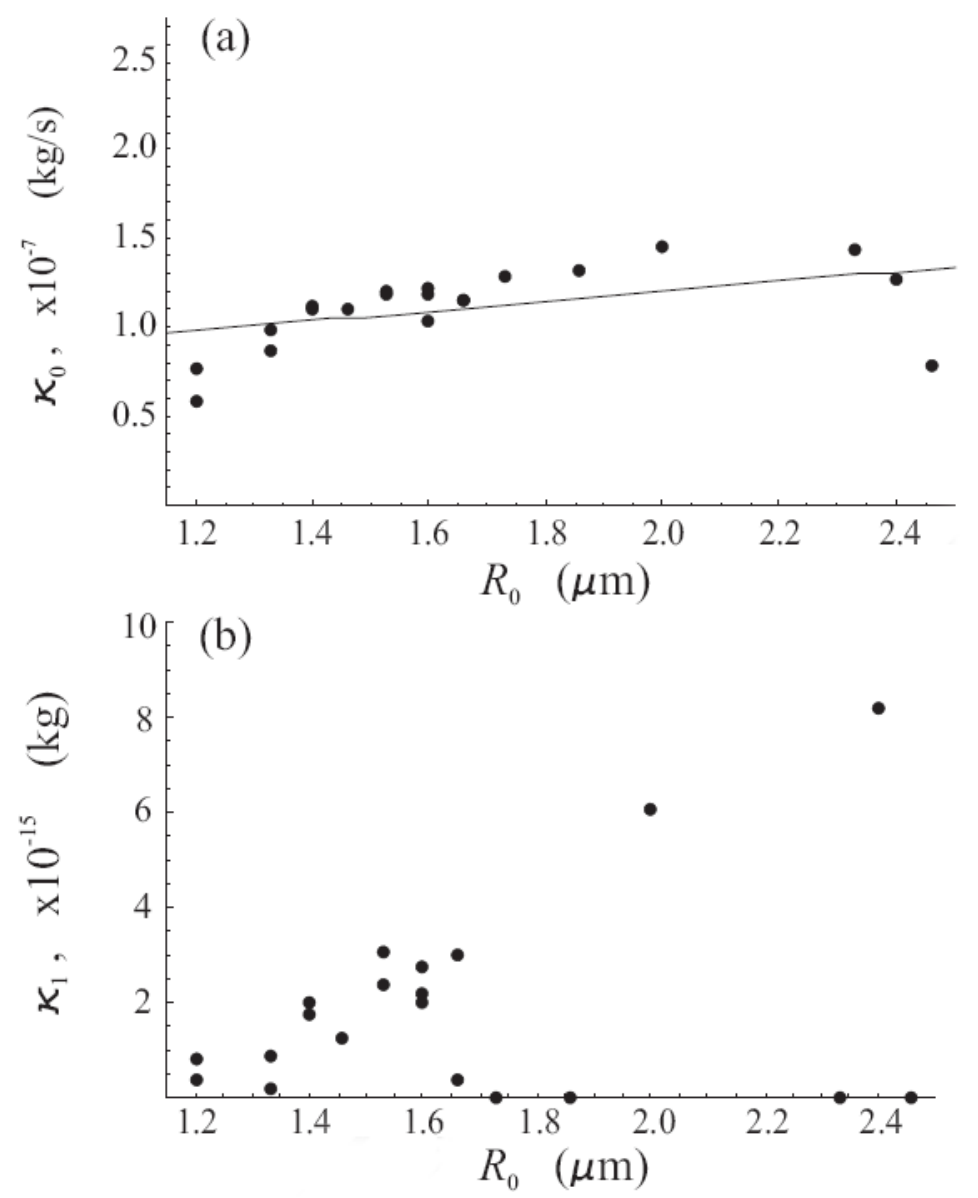

Fig. 6.

Best-fit values of the shell viscous coefficients $\kappa_{0}$ and $\kappa_{1}$ versus the initial bubble radius. The values of $\kappa_{0}$ and $\kappa_{1}$ were evaluated by the model with the Cross law, equation. (18). 